\title{
Listening time and the short-term perceptual deprivation effect*
}

\author{
JOSEPH R. LEVINE, ALICE PETTIT \\ and \\ BRUCE T. LECKART \\ California State University, San Diego, Calif. $92115 \dagger$
}

College students listened to randomly generated "melodies" until they felt they were "finished" listening. Preceding each melody, there was a perceptual deprivation interval (silence) of $1,14,28$, or $53 \mathrm{sec}$. Results indicate a direct relationship between duration of deprivation and listening time. Sensory adaptation interpretations were discussed and discarded. Results are interpreted in terms of arousal level notions.

\section{INTRODUCTION}

In a study by Leckart, Levine, Goscinski, \& Brayman (1970), Ss viewed moderately complex geometric figures which were preceded by short periods of perceptual deprivation. Duration of attention (looking time) was directly related to the duration of perceptual deprivation (darkness). The unique features of this experiment were: (1) unlike previous studies of sensory deprivation, the deprivation periods were very brief $(2-44 \mathrm{sec})$ rather than hours, and (2) perceptual deprivation was simply total darkness; i.e., elaborate procedures for eliminating or minimizing auditory and tactile stimulation were not employed. However, in both the long- and short-term deprivation studies, Ss display increased stimulus seeking or exploratory behaviors.

The authors therefore applied theoretical notions developed in the long-term deprivation situation to the short-term situation. Assuming an optimal level of arousal, as suggested by Hebb (1955), they argued that looking time "should" increase in order to compensate for the stimulus deficit incurred during the deprivation intervals.

An alternate explanation of their findings is that looking time increased after longer periods of deprivation because of greater levels of dark adaptation reached, and, therefore, longer periods of light adaptation, or "adjustment to the light," were required when the stimulus was presented before the Ss could even begin to "look at" the stimulus. A second alternative explanation is that Ss were matching or mimicking the duration of the dark periods.

The purpose of the present study is, first, to test the generality of the findings of Leckart et al (1970). It is reasonable to assume that if the stimulus-seeking explanation is correct, the duration of attention should increase with increased deprivation periods for the auditory modality as well as for the visual modality.

*The authors wish to thank Al Hillix and Robert Yaremko for their critical comments and valuable discussion of this research. +Formerly San Diego State College.
Further, the use of the auditory modality eliminates changes in the duration of attention attributable to deprivation intervals, because, for this modality, the deprivation periods would increase sensitivity or detectability of the subsequent stimulus rather than momentarily decreasing it. The hypothesis of the present experiment is that listening time increases as the duration of the silent intertrial interval increases.

\section{METHOD}

The Ss were 39 students at San Diego State College, who served in the experiment as part of a course requirement. Data from nine Ss were eliminated from the analysis because of machine failure or failure to follow instructions.

The stimuli were 20 "randomly generated" melodies, played on an electric organ and recorded on a 22-channel tape recorder (EFI audio notebook). The melodies were recorded on separate channels and were repeated without pause. Each of the melodies contained 17 tones between one octave above or below Middle C. There was an equal number of eighth, quarter, half, and full notes in each melody. All melodies concluded on Middle $C$ with a time value of one full note. The average intensity of the melodies was approximately $70 \mathrm{~dB}$.

The Ss were tested in a small lightproof chamber. They were told that the purpose of the experiment was to examine the effects of auditory stimuli on the galvanic skin response. Sham electrodes were attached to two fingers of the left hand, and the Ss were led to believe that the leads to the electrodes were connected to a polygraph in the adjacent cubicle. Ss were told to "listen to the tones until they were finished" and, when "finished," they pressed a button with their right hand. After instructing the Ss, the E fitted a set of EFI earphones on the Ss and then left the chamber. The Ss listened to the melodies in complete darkness. Four different interstimulus intervals (ISI), $1,14,28$, and $53 \mathrm{sec}$, were presented to each $\mathrm{S}$ five times.

The duration of the ISI (which was silent) was controlled by a set of automatic timers. The $\mathrm{E}$ selected channels on the audio notebook according to a predetermined schedule. A different random sequence of melodies and a different random sequence of ISIs were used for each $\mathrm{S}$.

\section{RESULTS}

The mean listening time following each ISI $(1,14,28$, and $53 \mathrm{sec}$ ) was calculated for each $\mathrm{S}$. These means are presented in Fig. 1. An analysis of variance indicated a significant difference in listening time as a function of the duration of the ISI $(F=9.98, \mathrm{df}=1 / 8, \mathrm{p}<.01)$. Inspection of Fig. 1 indicates that, as perceptual deprivation (ISI) increased from 1 to $53 \mathrm{sec}$, duration of attention (listening time) increased. The differences between the individual means were tested using a Duncan's multiple range test. The difference between the $1-\mathrm{sec}$ condition and all the other conditions, and the difference between the 14-sec and 53-sec conditions, was statistically significant $(\mathrm{p}<.05)$.

\section{DISCUSSION}

The results support the notion of a perceptual deprivation effect (PDE) in the auditory modality; i.e., listening time increased as a function of the duration of silent intervals. 


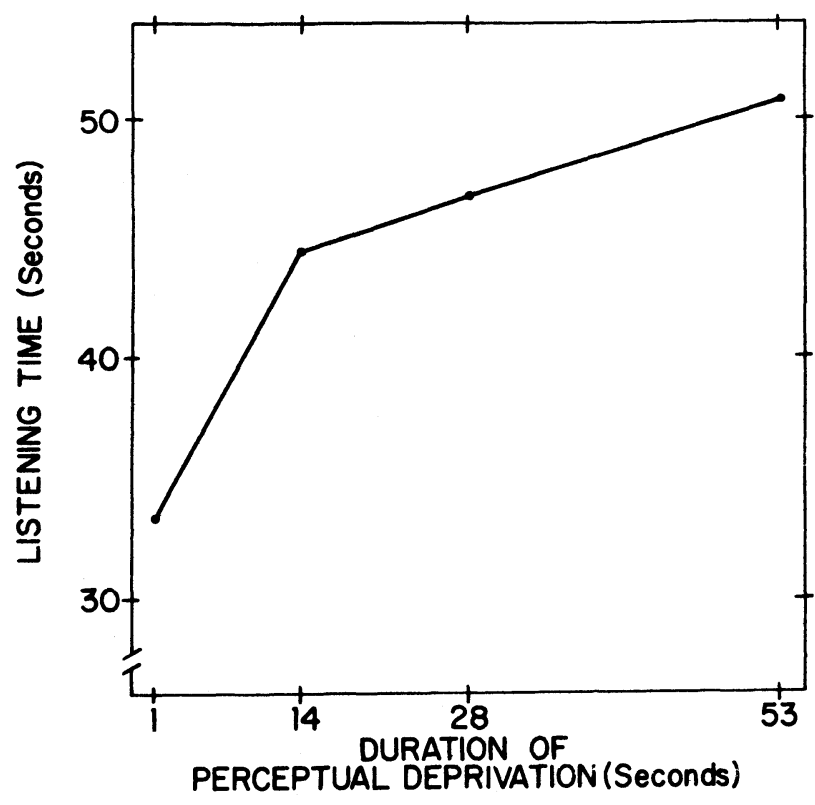

Fig. 1. Listening time as a function of previous deprivation.

Further, these results, in conjunction with the results of Yaremko, Glanville, Rofer, \& Leckart (1972), present convincing evidence that the PDE is not simply related to sensory adaptation or to the Ss' imitation of the duration of the preceding deprivation intervals.

An adaptation interpretation of the visual PDE is that during the longer deprivation intervals, the Ss dark adapted and, when the relatively bright stimulus was presented, a brief period of adjustment to the light was required. The added period of adjustment might account for the longer looking times subsequent to the longer deprivation intervals. However, in the present experiment two factors mitigate against an adaptation interpretation. First, the threshold shift resulting from the presentation of the stimuli would be approximately $30 \mathrm{~dB}$ (Lüscher \& Zwislocki, in Meuller, 1965) and the stimuli in the present study were well above that level, i.e., $70 \mathrm{~dB}$. Second, even the shortest silent intervals in the present study were sufficiently long so that complete recovery would be expected to occur within these intervals (Lüscher \& Zwislocki, as cited in Meuller, 1965). Finally, any residual adaptation effects would operate in the direction opposite to that predicted in the experimental hypothesis.

Leckart, Butler, \& Yaremko (1972) conducted an experiment in which half of the deprivation intervals contained stimulus slides. They found no systematic relationship between looking time and the duration of the preceding interval when the interval contained stimulus slides. They concluded that there was "... no evidence in support of the imitation hypothesis... [Leckart et al, 1972]." Furthermore, comparisons of the mean listening times in the present experiment and the looking time in the Leckart et al (1970) experiment cast further doubt on an imitation explanation. Both experiments employed similar deprivation intervals $(2,16,30$, and $44 \mathrm{sec}$ in the visual experiment and $1,14,28$, and $53 \mathrm{sec}$ in the auditory experiment), but the mean "exploratory" times are vastly different-12, 13.6, 14.3, and $14 \mathrm{sec}$ looking time and 34.3, $44.5,46.1$, and $50.9 \mathrm{sec}$ listening time. If Ss were in fact simply imitating the duration of the deprivation intervals, one would expect the exploratory times in both modalities to be similar.

The explanation of the PDE favored by the previous authors is based on the notion of maintenance of "optimum levels of arousal in a postulated neural center [Leckart et al, 1972]." According to this explanation, one of the determinants of arousal level is the amount of stimulus input. It is assumed that even short intervals of perceptual deprivation will result in a significant departure from the optimal level. The organism will attempt to restore his arousal level to the optimum by stimulus seeking or by exploratory behavior. It is further assumed that longer deprivation intervals yield greater departures from optimum that require longer exploratory periods for restoration of the arousal level.

It is also likely that the rate of stimulus input is a variable of considerable importance in determining the duration of attention subsequent to the deprivation intervals. Although rate was not manipulated directly in the present experiment, examinations of the results of the Leckart et al (1970) looking time data, the Yaremko et al (1972) tactile experiment, and the present listening time data support the hypothesis that, as rate increases, duration of attention decreases.

In the visual experiment, the entire stimulus scene is presented simultaneously to the $S$. The $S$ then scans the visual stimulus, changing the focus of his attention at his own pace. In the tactile task in which Ss are "feeling polygons," the entire stimulus is not "available" to the S simultaneously, as in the visual stimulus, although the rate of change of focus is still $S$ paced. The rate of change in the tactile situation is undoubtedly slower than that in the visual. Finally, in the auditory experiment, the rate of stimulus input is not under the $S$ 's control, but is determined by the character of the stimuli.

Comparison of the exploratory times of the three modalities, visual, tactile, and auditory, indicates that the rate of input is a powerful variable; i.e., the looking time durations are approximately three times shorter than the listening and feeling times.

\section{REFERENCES}

Hebb, D. E. Drives and the CNS (conceptual nervous system). Psychological Review, 1955, 62, 243-254.

Leckart, B. T., Levine, J. R., Goscinski, C., \& Brayman, W. Duration of attention: The perceptual deprivation effect. Perception \& Psychophysics, 1970, 7, 163-164.

Leckart, B. T., Butler, N., \& Y aremko, R. M. The looking time perceptual deprivation effect (PDE): A test of the imitation hy pothesis. Psychonomic Science, 1972, 26, 323-326.

Meuller, C. G. Sensory psychology. Foundation of Modern Psychology Series. Englewood Cliffs, N.J: Prentice-Hall, 1965.

Yaremko, R. M., Glanville, B., Rofer, C. P., \& Leckart, B. T. Tactile stimulation and the short-term perceptual deprivation effect. Psychonomic Science, 1972, 26, 89-90.

(Received for publication September 14, 1972.) 